\title{
Oral English Learning Strategies
}

\author{
Xiaoxue $\mathrm{Yu}$ \\ School of Foreign Languages, Changchun University of Science and Technology, China
}

\begin{abstract}
This thesis is concerned about the strategies of learning oral English. In the thesis, I introduce many strategies to help students to improve their oral English. Some of these strategies can directly influence their effectiveness of learning oral English such as cognitive strategies, but some do not have directly influence on oral English. The theory of individual differences is still another important one foe choosing effective strategies, which includes age, gender, learning style preferences, etc. In the last part, I carry out a SBI (strategies-based instruction) to test the effectiveness of the strategies I introduce. I get two major findings: one is that learning strategies do have effect on learning oral English; the other is that not a strategy is fit for all learners. The effectiveness of a strategy depends not only on what task it applies to but on personal characteristics.
\end{abstract}

\section{Index Terms - learning strategy, oral English learning strategy, individual difference, SBI}

Strategies of language learning are specific actions, behaviors, steps or techniques that students use to improve their progress in developing second language skills.(Maryam Khosravi, 2012) Oral English learning strategies are complex procedures that individuals apply to tasks, consequently, they may be represented as procedural knowledge which be acquired through cognitive associative and autonomous stages of learning, (Fateme Serri, Aliakbar Jafarpour Boroujeni \& Akbar Hesabi, 2012) in early stages of learning the strategies may be conscious and later be showed unconsciously. For most Chinese learners of English as a foreign language, it takes a great deal of psychological preparation and efforts to develop oral English communication skills. Oral fluency in English requires lowered inhibition, enhanced self-confidence, intrinsic motivation, greater ambiguity to tolerance, realistic personal goals and most of all, adventurous risk-taking.(Gai Fangpen \& Yang Dong, 2010) Meanwhile, effective communication also necessitates language accuracy based on less LI interference and pragmatic appropriateness obtained through both linguistic and non-linguistic media. In order to achieve the above two criteria of oral English, we should use efficient learning strategies. Next, I will introduce the oral English learning strategies one by one.

\section{APPlying INDIRECT STRATEGIES TO SPEAKING}

The indirect strategies are in favor of language learning. There are three branches. Metacognitive strategies allow learners to control their own cognition - that is, to coordinate the learning process by using functions as centering, arranging, planning, and evaluating. Affective strategies help to regulate emotions, motivations, and attitudes. Social strategies help students learn through interaction with others. All these strategies support and manage language learning without directly involving the target language. Indirect strategies are useful in virtually all language situations. (Rosna Awang Hashim, 1994)

\section{A. Metacognitive Strategies}

(a) Centering Your Learning (Maryam Khosravi, 2012)

First, paying attention. The strategy of paying attention is necessary for speaking. This strategy involves two modes, directed attention and selective attention. Directed attention means deciding generally or globally to pay attention to the task and avoid irrelevant distractions. In contrast, selective attention involves deciding in advance to notice particular details. Encourage directed attention by providing interesting activities and materials, reducing classroom distractions, reminding students to focus, and rewarding them when they do so. Facilitate selective attention by giving learners an incomplete chart to fill out, a table or checklist on which to mark details, or some other activity which requires attention to specifics.

Secondly, delaying speech production to focus on listening. This strategy relates to speaking. You do not have to teach or encourage this strategy, because many learners do it automatically by postponing their speaking in the target language for hours, days, weeks, or possibly even months. This phenomenon is often viewed as a way of focusing on listening comprehension before students feel comfortable enough to speak. The speech delay may be total or partial. The delay occurs because listening is more rapidly developed than speaking, and because speaking seems more threatening to many students. Some instructional theorists have stressed the importance of allowing a "silent period" for all learners, and various language teaching methods reflect this emphasis, but research evidence concerning the significance and optimal length of the silent period is mixed. Help build solid listening comprehension skills, and encourage students to speak as soon as they are ready, without any externally imposed delay.

(b) Planning and Arranging Language Learning.

First, understanding language learning. This strategy means uncovering what is involved in language learning. Learners often do not know much about the mechanics of language learning, although such knowledge would make 
them more effective learners. Books about language learning are a good source of information. Help your students by allowing them to talk about their language learning problems, ask questions, and share ideas with each other about effective strategies they have tried. Taking class time to talk about learning process will reap rewards for the students. This strategy can aid speaking.

Secondly, setting goals and objectives. Goals and objectives are expressions of students' aims for language learning. Students without aims are like boats without rudders; they do not know where they are going, so they might never get there! Goals and objectives should be noted in the language learning notebook, along with deadlines for accomplishing them and an indication as to whether those deadlines were met. Goals are generally considered to be long-range aims referring to the outcome of many months or even years. Objectives are short-term aims for hours, days, or weeks. Aid your students in determining goals and objectives in each of the skill areas, realizing that different students will have different aims.

Thirdly, identifying the purpose of a language task. This strategy involves determining the task purpose. The strategy of considering the purpose is an important one, because knowing the purpose for doing something enables learners to channel their energy in the right direction. Help your students understand the purpose by allowing them to discuss the purpose before doing the task itself. Figuring out the purpose for speaking is made easier by understanding the kind of speech being used-for instance, casual speech, deliberate speech, reading aloud from a written text, and speaking from a memorized script.

Lastly, seeking practice opportunities. Language learners must seek out - or create - opportunities to practice any and all of the four language skills. If students want to reach moderate to high proficiency, classroom time cannot usually provide adequate practice opportunities. Therefore, students will need to find additional chances to practice the language and must realize it is up to them to search for these occasions. This strategy underscores students' responsibility to generate their own opportunities to practice. Challenge your students to look for such chances whenever and wherever possible.

\section{(c) Evaluating Your Learning}

First, self-monitoring. This strategy does not center as much on using the language as it does on students' conscious decision to monitor - that is, notice and correct - their own errors in any of the language skills. Encourage your students to write down their most significant difficulties in their language learning notebooks and try to eliminate them. In considering a particular faux pas, learners can often benefit from trying to determine the reason why it is made. Self-monitoring is important for speaking, but students should not become obsessed with correcting every speech difficulty, because this would kill communication. Without expecting to be perfect, learners should notice and rectify their important speech problems, such as those which are socially offensive or which cause confusion.

Secondly, self-evaluating. This strategy involves gauging either general language progress or progress in any of the four skills including speaking. Global impressions are often faulty, and the more specific the learner is in self-evaluating, the more accurate the evaluation. Of course, any self-evaluation must take into consideration the difficulty of the situation or the language. Checklists, diaries, or journals can help learners evaluate their progress, at the same time as getting in touch with feelings. In speaking, there are many ways to self-evaluate. Learners may record their own speech on a tape recorder and then listen to the recording to find out how they sound compared with native speakers.

\section{B. Affective Strategies}

Affective strategies can regulate emotions, motivations, and attitudes. They cannot improve your speaking ability directly. They only can improve it indirectly.

\section{(a) Lowering Your Anxiety}

First, using progressive relaxation, deep breathing, or meditation. These techniques are all effective anxiety reducers, according to scientific feedback research. Progressive relaxation is that all the major muscle groups are alternately tense and relaxing. Deep breathing is often an accompaniment to progressive relaxation. It involves breathing low from the diaphragm, not just from the lungs. The simple act of deep breathing brings greater calmness almost immediately. Meditation means focusing on a mental image or sound to center one's thoughts, and it, too, helps to reduce the anxiety that often dogs language learners.

Secondly, using music. This strategy is useful before any stressful language task. Five or 10 minutes of soothing music can calm learners and put them in a more positive mood for learning. The language teaching method known as Suggestopedia is based partly on the use of baroque music to alter students' moods and mental states. The powerfully relaxation capabilities of music cannot be denied in the language learning context.

Lastly, using laughter. Laughter is the best medicine, as the saying goes. The use of laughter is potentially able to cause important biochemical changes to enhance the immune system, so many hospitals are now using "laughter therapy" to help patients relax. Language learners, too, can benefit from laughter's anxiety-reducing powers. Laughter brings pleasure to the classroom. Laughter is part of a general atmosphere of enjoyment for students of all ages.

\section{(b) Encouraging Yourself}

First, making positive statements. The strategy of making positive statements can improve each speaking skill. Make clear about the kinds of positive statements. Urge your students to say those statements regularly, especially before a potentially before a potentially difficult language activity. 
Secondly, taking risks wisely. This strategy involves a conscious decision to take reasonable risks regardless of the possibility of making mistakes or encountering difficulties. It also suggests the need to carry out this decision in action - that is, employing direct strategies to use the language despite fear of failure. This strategy does not imply wild, unnecessary risks, like guessing at random or saying anything at all regardless of its degree of relevance. Risk taking must therefore be tempered by good judgment. Deciding to be a wise risk taker may require the supportive use of other affective strategies, such as making positive statements or rewarding yourself.

\section{(c) Taking Your Emotional Temperature}

Listening to your body. One of the simplest but most often ignored strategies for emotion-assessment is paying attention to what the body says. Performance in speaking is affected by the learner's physical state. Negative feelings tighten the muscles and affect all the organs of the body. Positive feelings can have either a stimulating or a calming effect that is discernibly different from the effect of negative feelings. Language learners need to learn to pay attention to these physical sensations frequently.

\section{Social Strategies}

\section{(a) Asking Questions}

This strategy is mostly in speaking, because errors which are most obvious to other people occur in producing the new language. It is related to the strategy of self-monitoring, in which students notice and correct their own difficulties. In a spoken conversation, learners can ask the other person for correction of impatient problems-that is, those which cause confusion or offense. However, the other person cannot be expected to correct all errors made by the learner, because this would intimidate the learner, halt the conversation, and turn the conversation partner into a "speech cop"

\section{(b) Cooperating with Others}

First, cooperating with friends. This strategy involves a concerted effort to work together with other learners on an activity with a common goal or reward. Games, simulations, and other active exercises challenge students to develop their activity to cooperate with friends.

Secondly, cooperating with proficient users of the new language. (Rosna Awang Hashim, 1994) This strategy applies to speaking skill. When used for listening and speaking, this strategy involves taking specific steps to enhance communication with a proficient user of the new language.

\section{(c) Empathizing with Others}

First, developing cultural understanding. Background knowledge of the new culture often helps learners understand better what is heard in the new language. Such knowledge also helps learners know what is culturally appropriate to say aloud. Help students sharpen their culture understanding by injecting short cultural discussions into classroom activities, and by comparing and contrasting behavior in the students' native culture and the target culture. Outside of the classroom, encourage students to find out all they can about the target culture through reading, going to lectures, or watching films in the target language. All these activities develop greater cultural awareness, which is necessary for achieving proficiency in the new language.

Secondly, becoming aware of others' thoughts and feelings. Learners can purposefully become aware of fluctuations in the thoughts and feelings of particular people who use the new language. Such awareness makes learners be easier to close to the people they meet, helps them communicate each other more clearly, and suggests what to say and do.

Till now, we have discussed the indirect strategies. These strategies provide a rich and powerful support to any language learning effort. They work in concert with direct strategies.

\section{Factors Related to Second LANGUAGe LeARning Strategy USE}

Among recent studies regarding learning strategies, many have examined relationships between learning strategy use and learner variables such as motivation, gender, language proficiency, learning style, and learning beliefs.

First, age. Age has been found to be a factor affecting oral English strategies use. Older learners often use complex, sophisticated strategies.(Ehrman,M. \& Oxford,R.L, 1989)

Secondly, L2 proficiency. Several studies have indicated relationships between L2 proficiency and learner strategies. (Bialystok, E. 1981) used a questionnaire to gather data concerning the relationship between four strategies and second language proficiency. Functional practice and inferencing are two strategies concerned with learning resulting from communication in the target language. Formal practice and monitoring are two strategies used more consciously by learners to learn the target language. In investigating tenth graders and twelfth graders studying French, (Bialystok, E.1981) found that functional practice correlated significantly with second language proficiency in tenth graders, whereas functional practice, formal practice, and monitoring were related to L2 proficiency in twelfth graders,

Thirdly, learning styles. In synthesizing factors that influence the choice of learner strategies, asserted that learners" learning styles often determine the choice of L2 learning strategies".(Rebecca L.Oxford,1992), analytic-style learners tend to use strategies such as analyzing or separating words and phrases into parts, learning rules, and seeking similarities and differences between the target language and the first language. Globally-oriented learners prefer to use strategies such as guessing, scanning, and predicting to gain the gist of a passage and to read it holistically. Moreover, globally-oriented learners tend to use strategies such as paraphrasing and gesturing to converse with others in the target language in spite of limitations in linguistic knowledge. Visual learners are very likely to use 
strategies such as taking notes and writing word groups. On the other hand, auditory learners prefer to listen to tapes and practice aloud.(Ehrman,M. \& Oxford,R.L, 1989; Rebecca L.Oxford, 1992).

Fourthly, learning beliefs. Learners have different beliefs about language learning. Learning beliefs reflect learners' learning experiences and their socio-cultural educational backgrounds. Learners' learning beliefs, as researchers have suggested, play an important role in dictating learning strategy use. (Wenden, A, 1987) conducted semi-structured interviews with 25 adults enrolled part-time in an advanced level class at an American university. (Ellis, Rod, 2008) In this study, Wenden elicited subjects' views on language learning and examined the relationship between learners' learning beliefs and their choice of learner strategies. This study reported that learners recognized the importance in language use would often make use of communication strategies; that "learners who emphasized the importance of learning about the language tended to use cognitive strategies that helped them to better understand and remember specific items of language". Learners with beliefs in the use of language as often as possible, thinking in the target language, and living and studying in an environment in which the target language is spoken. Learners with beliefs in learning about the language emphasized learning grammar and vocabulary, learning from errors, enrolling in a formal course and being mentally active. (Nambiar, Radha, 2009)

Fifthly, gender. The study indicated gender differences in learning strategy use. Oxford and her colleagues conducted a series of studies on language learning strategies that(Hasan Bedir, 2010) examined relationships between learning strategy use and factors like motivation, academic major, and gender differences researched the relation between gender differences and strategies of language learning, and his study indicated that in many scopes women reported more on using strategies than did men. When Oxford and Nyikos conducted an analysis of the results of the SILL with 1200 university students through analysis of variance, they found that there were profound gender differences in strategy use and that female students reported using the following learning strategies more continually: formal rule-related practice strategies, general study strategies, and conversational input elicitation strategies. (Zhang Yan, 2012)

Lastly, motivation. (Gardner, R.C. 1985) In second language learning, motivation plays a significant part, and it can be divides into integrative motivation, in which the learner has a strong desire to recognize the target language, and instrumental motivation, in which the learner is motivated to learn the target language for utilitarian purposes. Although many research studies have examined relationships between motivation and achievement, only a few studies concern the relationship between motivation and learning strategies. Highly motivated students used more learning strategies and employed them more often than did less motivated students.(Rebecca Oxford \& M.Nyikos,1988) Ehrman and Oxford assessed the relationship between motivation and learning strategies by using the Affective Survey-a118-item, six-point Likert-scaled measure instrument developed by Ehrman and Oxford. Ehrman and Oxford reported that motivation was correlated with language learning strategy use from the, 20s to the high.503; that the highest correlation was between motivation and cognition strategies.

\section{EMPIRICAl Strategies-BASEd InStruction ON SPEAKING}

This part, I'll introduce the impact of strategies-based instruction on speaking English. It focuses on a study conducted at Changchun University. In China, English majors study hard in their four-year's study but they make different progress. The reason must be relevant to their English learning strategies. Therefore, I carried out a research into students' English learning strategies. My investigation was mainly based on self-report surveys.

The objects of my research were the English majors in Grade One at college. As to the students, they had mastered 2500 English words and expressions. Thirty students were singled out from English department in Changchun University. In March, 2013, the students finished the papers and gave the answers which were just students' personal opinions.

The items were designed to assess how the students went about learning English. Each represents a particular kind of language learning behavior. The results would help to know more about students' English learning.

The student who chose "a" would get one point, chose "b" would get two points, chose "c" would get three points, chose "d" would get four points . After calculating, I got the result shown in the below table:

\begin{tabular}{ll}
\hline Score Group & Number of Students \\
\hline$<20$ & 1 \\
$20-30$ & 7 \\
$30-40$ & 14 \\
$40-50$ & 6 \\
$50-60$ & 2 \\
\hline
\end{tabular}

The thirty students' scores were found from 56 down to 18 . Among them, one was below 20 points. Seven were between 20 and 30 points. Fourteen were in the range from 30 to 40 points. Six were in the range from 40 to 50 . Only 2 students got above 50 points. 1 summed all their scores up and got 1070 . Then I divided 1070 by 30 , obtaining 35.7. And dividing 35.7 by 15,1 got the overall average score for each item. It was 2.4 points.

The number indicated that the students used learning strategies on rare occasions when they were learning English. The statistics also suggested that it was very necessary for them to master some language learning strategies. 
According to the three different tasks, students completed the strategies checklists which indicated data on strategy using at 3 points: The first task was before speaking, the second task was during itself, the last one was after the completion. The checklists were intended to put three stages into strategy using: firstly, prepare to use the language skill, secondly self-supervision to use the skill and thirdly self-reflection after using the skill. The strategies checklists reflected both those found in the language strategies' literature, as well as those particular to the three tasks.

After test the checklists, there were some questions to do self-reflection. During completed the three tasks, some questions handled the students' experiences. Another question determined whether students as language learners were more independent in the six weeks language study.

The English native speaker didn't know whether replied the 3 tasks when they assessed students' tapes in English. There were two taped sample, one was experimental group, another was comparison group.

Students made the spoken language which was assessed three aspects. Firstly, showed confidence in communication. Secondly, made correction in grammar. Thirdly, have control on vocabulary.

There are two aspects to assess the task of retelling story: one is to identify the key elements. Another is to appropriate ordering of these elements.

After the rating the tapes, the native speaker gave every tape a score. Then, I compared the two groups' scores to find out which group was better in speaking proficiency. In this step, I used the statistical method and counting percentage method. I computed out how many percent the students of over 90 occupied the whole students both of the experimental group and of comparison group for every topic. Then the percents of the students of over 80 , over 70 , over 60 and below 60 in the two groups. I got the following tables:

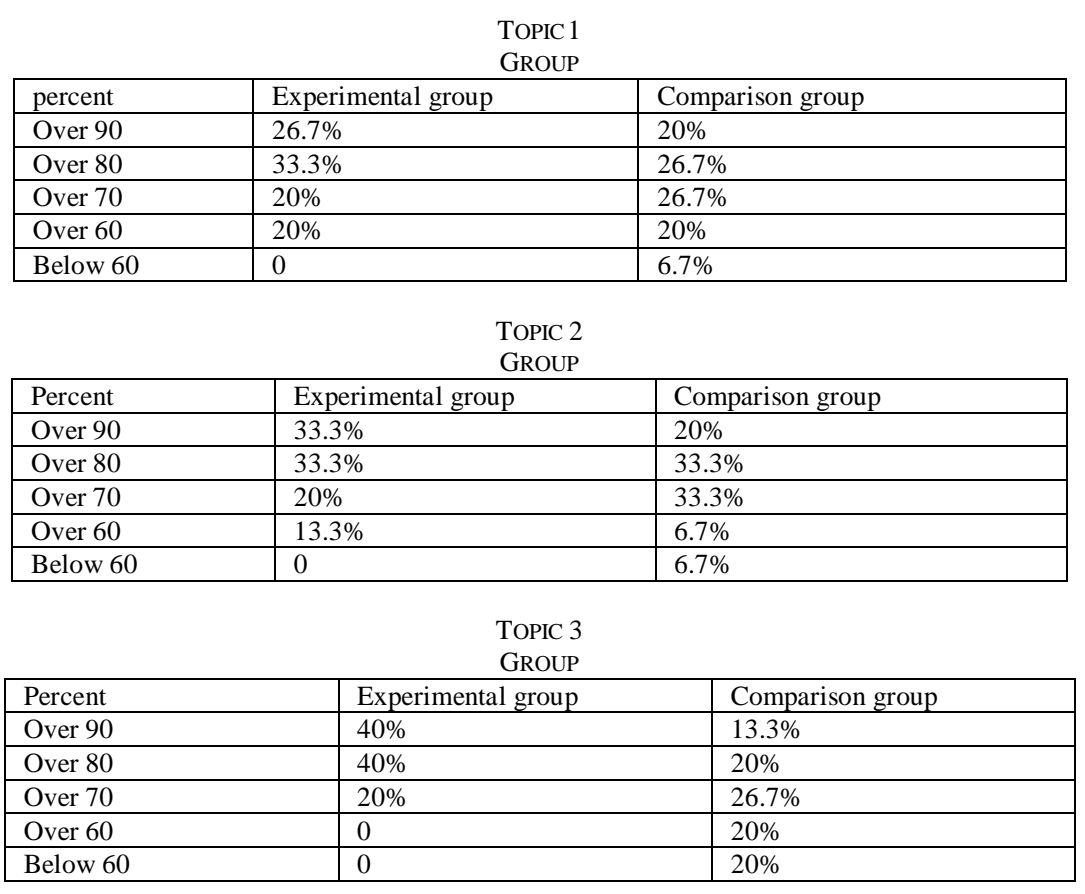

After studying these three tables, we can see the experimental group students did better than the comparison group students on every topic especially the difference in topic three was more clearly. It proved that strategies-based instruction had effects on improvement of speaking proficiency. And the strategies did have effects on English learning. According to their remarks, I knew that some strategies which were made by the teacher and the students of experimental group did not suit some students, on the contrary, these strategies suited other students. So strategies vary with different person.

In this study, I got major findings. Thirty college students were participated in a SBI action. They were comparison students getting six weeks language study. The students completed questionnaire. After three tasks strategy checklists were also finished.SBI to test the strategies was effectively.

We could add preparatory strategies and monitoring strategies to one or more aspects for the experimental group students. We note that these were primarily language use strategies, employed in order to help retrieve language material, to rehearse it, and to use it communicatively. In addition, several of the strategies used increasingly could be seen as language learning strategies. Translating specific words and practicing their pronunciation in preparation for speaking were learning strategies if as a result of using them the learners became more cognizant of grammatical relationships, since increases in the use of those two strategies correlated significantly with an increase in grammar ratings.

We could add some strategies to task performance for the comparison group. The correlation between creating a mental picture and a higher rating on vocabulary could be viewed as a learning strategy, since mental imagery helps in 
the storage and retrieval of new lexical items, most of the significant correlations seem to involve language use strategies, such as increased attention to pronunciation and the use of paraphrase being linked to an increased vocabulary rating, and an increase in positive self-talk being significantly correlated with a higher self-confidence rating. All three of these - attention to pronunciation, use of paraphrase, and positive self-talk-could be seen as communication strategies par excellence.

Instances such as these, where comparison students had positive correlations and experimental students did not, would seem to run counter to expectation, since the strategies were emphasized in the treatment. A possible interpretation for these findings is that some experienced speakers can make use of strategies without many strategy indications.

After discussion with the students of experimental group, the instructor and the native speaker of English, we got a conclusion that individual differences play very important role in strategy choosing and they directly influence the effects of strategies.

\section{CONCLUSION}

From my SBI (strategies-based instruction), we can see that after taking the instructed strategies, the scores of experimental group have evidently improved, but the scores of comparison group which didn't take any learning strategies haven't improved evidently.

Learning strategies have effectiveness on oral English learning. We should try our best to exert as many learning strategies as we can on our oral English learning. Before teaching oral English, teachers should introduce some learning strategies to the students first, then the importance of learning strategies. Individual differences also influence on learning strategy choice. Teachers, of course, should pay enough attention to students' personal differences such as gender, age, motivation, learning style, learning beliefs etc. So to different students, teachers should introduce different strategies to them in order to get the best learning result. Most important of all, while teaching oral English, teachers also should apply these learning strategies to their teaching in order that students can use them automatically because the students can clearly see the improvement of oral English level. In all, learning strategies really can improve your learning results. In practicing oral English, utilizing learning strategies also can greatly improve your oral English level.

\section{REFERENCES}

[1] Bialystok, E. (1981). The role of linguistic knowledge in second language use. Studies in Second Language Acquisition, Vol 4, No, 1, 31-45.

[2] Ehrman, M. \& Oxford, R.L. (1989). Effects of sex differences, career choices and psychological type on adult learning strategies. Modern Language Journal, Vol 73, No. 1, 1-13.

[3] Ellis, Rod. (2008). Learner Beliefs and Language Learning. Asian EFL Journal, Vol 10, No 4, 7-25.

[4] Fateme Serri, Aliakbar Jafarpour Boroujeni \& Akbar Hesabi. (2012). Cognitive, Metacognitive, and Social/Affective Strategies in Listening Comprehension and Their Relationships with Individual Differences. Theory and Practice in Language Studies, Vol 2, No 4, 843-849

[5] Gai Fangpeng \& Yang Dong. (2010). A Study on College Students' Anxiety to Spoken English. Canadian Social Science, Vol 6, No 2, 95-101.

[6] Gardner, R.C. (1985). Social Psychology and Second Language Learning: The role of attitudes and motivation. London: Edward Arnold.

[7] Hasan Bedir. (2010). Teachers' beliefs on strategies based instruction in EFL classes of young learners. Procedia - Social and Behavioral Sciences, Vol 2, No. 2, 5208-5211.

[8] Hu Yanfeng. (2009). A Survey on the English Learning Strategy of the Rural High School Students. English Language Teaching, Vol 2 No.3, 232-236.

[9] Maryam Khosravi. (2012). A Study of Language Learning Strategies Used by EFL Learners in Iran: Exploring Proficiency Effect on English Language Learning Strategies. Theory and Practice in Language Studies, Vol 2, No 10, 2122-2132.

[10] Nambiar, Radha. (2009). Learning Strategy Research -- Where Are We Now. Reading Matrix: An International Online Journal, Vol 9, No 2,132-149.

[11] Rebecca Oxford \& M.Nyikos. (1988). Vive la difference? Reflections on Sex differences in use of language learning strategies. Foreign Language Annals, Vol 21, No.4, 321-329.

[12] Rebecca L. Oxford. (1992). Research on Second Language Learning Strategies. Annual Review of Applied Linguistics, Vol 13, No.3, 174-187.

[13] Rosna Awang Hashim. (1994). Examining Learners' Language Learning Strategies. RELC Journal, Vol 25, No. 2, 1-20.

[14] Wenden, A. (1987). Learner Strategies for Learner Autonomy. New York: Prentice Hall.

[15] Zhang Yan. (2012). The Impact of Listening Strategy on Listening Comprehension. Theory and Practice in Language Studies, Vol 2, No 3, 625-629.

Xiaoxue Yu was born in Changchun City, Jilin Province, China, in 1986. She received her Bachelor's Degree in Literature from Beihua University, China in 2009.

She is currently a postgraduate in School of Foreign Languages, Changchun University of Science and Technology, China. Her research interests include linguistics and American literature. 